\title{
Precise Spatial Relationships between Mossy Fibers and Climbing Fibers in Rat Cerebellar Cortical Zones
}

\author{
Angelique Pijpers, ${ }^{1}$ Richard Apps, ${ }^{2 \star}$ Joanne Pardoe, ${ }^{2}$ Jan Voogd, ${ }^{1}$ and Tom J. H. Ruigrok ${ }^{1 \star}$ \\ ${ }^{1}$ Department of Neuroscience, Erasmus Medical Center Rotterdam, 3000 DR Rotterdam, The Netherlands, and ${ }^{2}$ Department of Physiology, University of \\ Bristol, BS8 1TD Bristol, United Kingdom
}

Classically, mossy fiber and climbing fiber terminals are regarded as having very different spatial distributions in the cerebellar cortex. However, previous anatomical studies have not studied these two major cerebellar inputs with sufficient resolution to confirm this assumption. Here, we examine the detailed pattern of collateralization of both types of cerebellar afferent using small injections of the bidirectional tracer cholera toxin b subunit into the posterior cerebellum. The cortical and zonal location of these injections was characterized by mapping climbing fiber field potentials, the distribution of retrogradely labeled olivary neurons, and the intrinsic zebrin pattern of Purkinje cells.

Labeled climbing fiber collaterals were distributed as longitudinal strips and were always accompanied by clusters of labeled mossy fiber rosettes in the subjacent granular layer. Two- and three-dimensional reconstructions and quantitative analysis showed that mossy fibers also collateralized to other stripe-like regions usually below Purkinje cells with the same zebrin-positive or zebrin-negative characteristics as that of the injection site and associated climbing fiber collaterals. The distribution of retrogradely labeled neurons in two major sources of mossy fibers, the lateral reticular and basilar pontine nuclei, revealed interlobular and some interzonal differences.

These data indicate that nonadjacent cerebellar zones, sharing the same climbing fiber input and zebrin identity, also share a common mossy fiber input. Other cerebellar cortical regions that receive collaterals from the same mossy fibers usually also have the same zebrin signature. Together with the distribution of neurons in precerebellar centers, the findings suggest a revision of the modular hypothesis for information processing in the cerebellar cortex.

Key words: Purkinje cell; inferior olive; zebrin; cerebellar modules; mossy fiber rosette; cholera toxin b subunit

\section{Introduction}

The two major afferent systems of the cerebellum, the climbing fiber and mossy fiber systems, are characterized by marked morphological, anatomical, and physiological differences (Ito, 1984). Neurons from specific parts of the inferior olive send their climbing fibers to narrow, longitudinally arranged, strips of Purkinje cells (Voogd and Glickstein, 1998; Sugihara and Shinoda, 2004). These strips of Purkinje cells project to specific regions of the cerebellar nuclei that also receive direct input from collaterals of the same climbing fibers, thus resulting in a modular arrangement of olivo-cortico-nuclear connections (Pijpers et al., 2005). Because the cerebellar nuclei constitute the output of the cerebellum, these modules are considered to act as functional entities (Voogd and Bigaré, 1980; Apps and Garwicz, 2005).

\footnotetext{
Received Nov. 18, 2005; revised Sept. 27, 2006; accepted 0ct. 13, 2006.

This work was supported by the Netherlands Organization for Scientific Research, Division of Earth and Life Sciences (Project Number 810.37.005) and the Dutch Ministry of Health, Welfare, and Sports. R.A. was supported by a Senior Research Fellowship funded by the Medical Research Council (United Kingdom). We thank R. Bissett, C. Everard, E. Sabel-Goedknegt, J. van der Burg, and E. Dalm for their technical assistance.

${ }^{*}$ R.A. and T.J.H.R. are joint senior authors.

Correspondence should be addressed to Tom J. H. Ruigrok, Department of Neuroscience, Erasmus Medical Center Rotterdam, P.0. Box 1738, 3000 DR Rotterdam, The Netherlands. E-mail: t.ruigrok@erasmusmc.nl.

J. Pardoe's present address: Psychiatry Centre of Excellence for Drug Discovery, GlaxoSmithKline, New Frontiers Science Park, CM195 5AW Harlow, Essex, UK.

DOI:10.1523/JNEUROSCI.2905-06.2006

Copyright $\odot 2006$ Society for Neuroscience $\quad$ 0270-6474/06/2612067-14\$15.00/0
}

The organization of the mossy fibers, which synapse with granule cells and local cortical interneurons, appears much less well structured. They originate from many parts of the CNS such as the pontine nuclei, trigeminal complex, lateral reticular nucleus, dorsal column nuclei, and the spinal cord (Yatim et al., 1996; Alisky and Tolbert, 1997; Serapide et al., 2001; Odeh et al., 2005). Individual mossy fibers are characterized by a divergent pattern of projections (i.e., they distribute collaterals along both the rostrocaudal and mediolateral axes) (Mihailoff, 1993; Wu et al., 1999; Sultan, 2001; Ruigrok, 2003). The wide divergence of mossy fiber-based information is further magnified by the granule cell-parallel fiber system.

The organizational differences between the mossy fiber/parallel fiber system and the climbing fiber system have been central to all major theories of cerebellar function (Marr, 1969; Albus, 1971; Ito, 1984). Nevertheless, the anatomical interrelationship between mossy and climbing fiber systems has not, so far, been studied with sufficient detail to evaluate the structural relationships underpinning these hypotheses. Such an investigation should also provide an anatomical basis for understanding recent physiological observations on receptive field characteristics of climbing fibers and mossy fibers (Brown and Bower, 2001; Ekerot and Jorntell, 2001; Apps and Garwicz, 2005; Ito, 2006).

We have therefore charted the detailed topographical relationship of mossy and climbing fiber afferents by analyzing the 
Table 1. Summary of cases described in text and/or shown in figures

\begin{tabular}{|c|c|c|c|c|c|c|c|c|c|c|c|c|c|c|}
\hline \multirow[b]{2}{*}{ Case } & \multirow[b]{2}{*}{ Physiology } & \multicolumn{4}{|c|}{ I0 labeling } & \multirow[b]{2}{*}{ Anatomical zone } & \multirow[b]{2}{*}{ Zebrin } & \multicolumn{3}{|c|}{ LRn labeling } & \multicolumn{3}{|l|}{ Pn labeling } & \multirow[b]{2}{*}{ Ratio Pn/LRn } \\
\hline & & MAO & vfDA0 & $\mathrm{PO}$ & Total & & & Ipsilateral & Contralateral & Total & Ipsilateral & Contralateral & Total & \\
\hline GR03 & No & 0 & 167 & 0 & 167 & C1-COP & P4-(e) & 358 & 100 & 458 & 20 & 778 & 798 & 1.74 \\
\hline 979 & No & 8 & 77 & 4 & 89 & C1-PMD & $\mathrm{P} 5 \mathrm{a}-$ & 241 & 70 & 311 & 115 & 594 & 709 & 2.28 \\
\hline $\mathrm{APO}^{a}$ & FL-ip & 0 & 104 & 1 & 105 & C1-PMD & P5a- & 181 & 53 & 234 & 82 & 550 & 632 & 2.70 \\
\hline AP10 & FL-ip & 0 & 73 & 0 & 73 & C1-PMD & P5a- & 96 & 18 & 114 & 33 & 355 & 388 & 3.40 \\
\hline 981 & No & 23 & 1 & 0 & 24 & $\mathrm{C} 2-\mathrm{COP}$ & $\mathrm{P} 5+/ 7+$ & 59 & 14 & 73 & 17 & 259 & 276 & 3.78 \\
\hline AP09 & FL-ip, FL-co & 37 & 3 & 0 & 40 & C2-PMD & P5+ & 103 & 32 & 135 & 39 & 573 & 612 & 4.53 \\
\hline 864 & No & 93 & 4 & 0 & 97 & C2-PMD & $\mathrm{P} 5+$ & 63 & 26 & 89 & 58 & 432 & 490 & 5.51 \\
\hline A41 & Face-ip & 29 & 0 & 0 & 29 & C2-Crus2B & P5+ & 36 & 17 & 53 & 52 & 337 & 389 & 7.34 \\
\hline 978 & No & 5 & 0 & 12 & 17 & D0/D1-PMD & $P 6+/ 7+$ & 37 & 10 & 47 & 13 & 446 & 459 & 9.77 \\
\hline $\mathrm{APO}^{a}$ & FL-ip & 0 & 6 & 53 & 59 & D0/D1-PMD & $P 6+/ 7+$ & 53 & 18 & 71 & 42 & 1051 & 1093 & 15.39 \\
\hline 980 & No & 0 & 0 & 20 & 20 & D1-PMD & $P 6+/ 7+$ & 19 & 9 & 28 & 20 & 427 & 447 & 15.96 \\
\hline
\end{tabular}

Cell counts were performed in a one of four series of sections in all cases. co, Contralateral; FL, forelimb; 10 , inferior olive; ip, ipsilateral.

${ }^{a}$ Experiments were performed in Bristol, United Kingdom; all other experiments were conducted in Rotterdam, The Netherlands.

distribution of their collateral terminations as labeled from small injections of cholera toxin b subunit (CTb) into specific climbing fiber-defined zones of the posterior cerebellar cortex. In addition, these patterns were related to the intrinsic bands of zebrinpositive and -negative Purkinje cells (Hawkes and Leclerc, 1987; Voogd et al., 2003). We show that labeled mossy fiber collaterals are always present in the granular layer directly below labeled collaterals of climbing fibers. In addition, mossy fibers also terminate in strip-like clusters at other cortical locations. Twodimensional (2D) and three-dimensional (3D) reconstructions and quantitative analysis show that these locations may be functionally linked to the site of injection because they often have the same zebrin signature. These findings, in combination with analyses of the distribution of labeled neurons in two major brainstem sources of mossy fibers, suggest that the mossy fiber system makes use of highly organized projection patterns that relate closely to the topography of the climbing fiber system.

\section{Materials and Methods}

Eleven adult, male Wistar rats (weight, 200-300 g) were used for the present study (Table 1$)$. Most experiments $(n=9)$ were performed at the Department of Neuroscience, Erasmus Medical Center Rotterdam, in accordance with the National Institutes of Health Guide for the Care and Use of Laboratory Animals and with permission from the national committee overseeing animal experiments. In three of these experiments, the injections were guided by electrophysiological recording and the others $(n=6)$ were guided by anatomical landmarks alone (Table 1$)$. The initial stage of the two additional experiments in which the injections were guided by electrophysiological recording, was performed at the Department of Physiology, University of Bristol in accordance with the United Kingdom Animals (Scientific Procedures) Act, 1986, and was approved by the institutional animal license advisory group. Six additional cases from Bristol and two from Rotterdam were consulted, but their injection sites were found to involve multiple cerebellar cortical zones and were not included in the present paper.

Surgical procedures. In the electrophysiologically guided experiments, animals were initially anesthetized by an intraperitoneal injection of 60 mg/kg pentobarbital (Sagatal; Rhône Merieux, Harlow, UK; or Nembutal; Ceva Sante Animale B.V., Maasluis, The Netherlands). In all other cases, animals were anesthetized by an intraperitoneal injection of a ketamine/xylazine mixture (40-60 mg/kg and $3 \mathrm{mg} / \mathrm{kg}$; ketamine; Alfasan, Woerden, The Netherlands; xylazine; Rompun, Bayer AG, Leverkusen, Germany). In all experiments, supplementary doses were given intraperitoneally to maintain a deep level of anesthesia as monitored by absence of the pinch withdrawal reflex and loss of muscle tone. Body temperature was maintained and regulated within physiological limits with a thermostatically controlled heating blanket $\left(37 \pm 1^{\circ} \mathrm{C}\right)$. All animals were placed in a stereotaxic head holder and a small craniotomy exposed part of the posterior lobe of the cerebellum on one side, allowing access to crus
2, paramedian lobule (PMD), and copula pyramidis (COP). The atlantooccipital membrane and the dura were opened and the exposed cerebellar surface was kept moist by periodic flushing with sterile saline solution.

In experiments guided by electrophysiology $(n=5)$, evoked climbing fiber field potentials were recorded extracellularly with glass-coated tungsten microelectrodes (tip, $\sim 50 \mu \mathrm{m}$; impedance, $50-140 \mathrm{k} \Omega$ ). The responses were recorded on the cerebellar surface as the result of brief $(0.1 \mathrm{~ms})$ electrical percutaneous stimulation of the ipsilateral or contralateral forelimb or hindlimb (for a detailed description, see Atkins and Apps, 1997). In these experiments, the paravermal part of PMD, COP, or crus $2 \mathrm{~b}$ was mapped at $0.1 \mathrm{~mm}$ intervals in a mediolateral direction. In this way, the $\mathrm{C}$ zones located within these lobules were electrophysiologically identified. Field potentials were amplified and bandpass filtered (30 $\mathrm{Hz}$ to $5 \mathrm{kHz}$ ), and a Humbug device (Quest Scientific, North Vancouver, British Columbia, Canada) was used to eliminate $50 \mathrm{~Hz}$ electrical interference. Evoked cerebellar responses were recorded and stored for offline analysis with customized trigger-sampled software (Spike 2; Cambridge Electronic Design, Cambridge, UK) running a Cambridge Electronic Design 1401 plus A/D converter (Pardoe and Apps, 2002). After localization of the desired cortical zone by mapping response amplitude, an iontophoretic injection ( $4 \mu \mathrm{A}$ anodal, $7 \mathrm{~s}$ on, $7 \mathrm{~s}$ off, $10 \mathrm{~min}$ ) was made of cholera toxin b subunit $(\mathrm{CTb})(1 \%$ in $0.1 \mathrm{M}$ phosphate buffer, pH 7.4; List Biological Laboratories, Campbell, CA) using a glass micropipette (tip, $10-15 \mu \mathrm{m}$ ) attached to a custom-made iontophoresis device (Ruigrok et al., 1995; Teune et al., 1998). Microinjections were made at a depth of $200-300 \mu \mathrm{m}$ at sites where Purkinje cell activity could be recorded. This procedure was followed for cases AP02, AP03; AP09, AP10, and A41. The remaining six experiments (cases 864, 978, 979, 980, 981, and GR03) were performed in Rotterdam using cerebellar surface coordinates [derived from Atkins and Apps (1997)] to guide the iontophoretic CTb injections.

In all experiments, the micropipette was withdrawn after the microinjection, the overlying muscle and skin layers were sutured, and the animal was allowed to recover. Postoperative analgesia was provided by subcutaneous administration of buprenorphine $(0.05-0.1 \mathrm{mg} / \mathrm{kg}$; Temgesic; Schering-Plough, Union, NJ). In cases in which increased fluid excretion led to an audible respiration during surgery, atropine sulfate $(0.6 \mathrm{mg} / \mathrm{kg}$; Atrocare; Animalcare, Dunnington, UK) was administered subcutaneously. All animals recovered uneventfully and were monitored daily.

Histology. After a $7 \mathrm{~d}$ survival period, animals were killed by means of transcardial perfusion under deep barbiturate anesthesia (100-200 mg/ $\mathrm{kg}$, i.p.). An initial flush of $500 \mathrm{ml}$ of $0.9 \%$ saline in $0.01 \mathrm{M}$ phosphate buffer, $\mathrm{pH} 7.2-7.4$ (PB) was given, followed by $1000 \mathrm{ml}$ of fixative ( $4 \%$ paraformaldehyde, $0.1 \%$ glutaraldehyde, and $4 \%$ sucrose in $0.05 \mathrm{M} \mathrm{PB}$. The brain was removed from the skull and postfixed for $1-2 \mathrm{~h}$, after which it was rinsed and stored overnight in $0.05 \mathrm{~m} \mathrm{~PB}$ containing $10 \%$ sucrose $\left(\right.$ at $\left.4^{\circ} \mathrm{C}\right)$, and subsequently embedded in $11 \%$ gelatin, $10 \%$ sucrose. Cerebellar and caudal brainstem blocks were hardened in $10 \%$ formalin, $30 \%$ sucrose solution for $3 \mathrm{~h}$ and were stored overnight in 0.05 M PB containing $30 \%$ sucrose at $4^{\circ} \mathrm{C}$. 
The tissue blocks were sectioned transversally at $40 \mu \mathrm{m}$ on a freezing microtome and collected serially in eight glass vials containing $0.05 \mathrm{M} \mathrm{PB}$ (Ruigrok, 2003). Every other vial was processed either for CTb immunohistochemistry or for a combined CTb-zebrin immunostaining; the four remaining vials served as spares. Before processing, free-floating sections were rinsed three times in PBS (0.05 $\mathrm{M} \mathrm{PB}, 0.9 \% \mathrm{NaCl}, \mathrm{pH} 7.6)$ and were subsequently incubated for $48-72 \mathrm{~h}$ in a polyclonal anti-choleragenoid raised in goat (goat anti-CTb; lot 703; List Biological Laboratories) diluted 1:15,000 in PBS and containing 0.5\% Triton X-100 (PBS+, pH 7.4) at $4^{\circ} \mathrm{C}$ under constant gentle stirring. After subsequent rinsing in PBS, sections were further incubated in biotinylated donkey anti-goat (1:2000; Jackson ImmunoResearch, West Grove, PA; in PBS +) for 1-2 h at room temperature (RT), rinsed in PBS, and incubated for 1-2 h (RT) in avidinbiotin complex (ABC Elite; Vector Laboratories, Burlingame, CA) in PBS + . After an additional rinse in $0.05 \mathrm{M} \mathrm{PB}$, two vials of sections were processed for $\mathrm{CTb}$ labeling using 3,3'-diaminobenzidine tetrahydrochloride (DAB) as the chromogen $\left(0.25 \% \mathrm{DAB}\right.$ and $0.005 \% \mathrm{H}_{2} \mathrm{O}_{2}$ in 0.05 м $\mathrm{PB}, \mathrm{pH} 7.2$, for $20 \mathrm{~min}$ ), resulting in a brown reaction product. The other two vials were incubated in DAB-cobalt for $10-15 \mathrm{~min}$ [ $1.5 \mathrm{ml}$ of $\mathrm{CoSO}_{4}(1 \%)$ and $1.5 \mathrm{ml}$ of $\mathrm{NiSO}_{4}(1 \%)$ added to the DAB solution], resulting in a black reaction product. All vials were then rinsed in $0.05 \mathrm{M}$ $\mathrm{PB}$. The two vials of sections also processed for zebrin staining were rinsed in PBS and free-floating sections were incubated for $48-72 \mathrm{~h}$ in zebrin II antibody [1:150 in PBS+ containing 2\% normal horse serum (NHS) at $\left.4^{\circ} \mathrm{C}\right]$. The zebrin II antibody, which was kindly provided by Dr. R. Hawkes (University of Calgary, Calgary, Alberta, Canada), was produced by immunization with a crude cerebellar homogenate of the weakly electric fish Apteronotus (Brochu et al., 1990). After rinsing in PBS, sections were incubated for $2 \mathrm{~h}$ in rabbit anti-mouse HRP (p260; DakoCytomation, High Wycombe, UK; 1:200 in PBS+, containing 2\% NHS). Subsequently, the sections were thoroughly rinsed in $0.05 \mathrm{M} \mathrm{PB}$, again processed (this time without heavy metal ions) with $\mathrm{DAB}$ and $\mathrm{H}_{2} \mathrm{O}_{2}$ (15-20 min), and rinsed in $0.05 \mathrm{M} \mathrm{PB}$. All sections were mounted serially on slides in a chromic alum solution, air dried, and counterstained with thionin, dehydrated in graded alcohol and xylene, and finally coverslipped with Permount.

Analysis. In selected cases, in which the injection site was restricted primarily [in some cases entirely (Table 1)] to one cerebellar cortical zone, as judged by the distribution of retrogradely labeled cells in the inferior olive, sections were examined and plotted using an Olympus (Tokyo, Japan) microscope fitted with a Lucivid miniature monitor and Neurolucida software (MicroBrightField, Colchester, VT). A complete series of one in four transverse sections (interval, $160 \mu \mathrm{m}$ ) was examined for labeled climbing fiber collaterals and mossy fiber rosettes in the cerebellar cortex. The outline of each cerebellar section was plotted with a $2.5 \times$ objective, whereas mossy and climbing fiber terminals were examined using a $20 \times$ objective and the motorized stage scan option of the plot program. A $40 \times$ objective was occasionally used if there was any doubt concerning the identity of a labeled structure. Labeled neurons within the inferior olive were also plotted using a one in four series of transverse sections ( $160 \mu \mathrm{m}$ interval), resulting in a series of $14-16$ plots. Cell labeling was assigned to different olivary subdivisions based on the standard diagrams of Ruigrok and Voogd (2000). Olivary areas containing labeled neurons were also indicated in standardized flattened surface maps of the individual subnuclei (Ruigrok, 1997; Ruigrok and Voogd, 2000). Similarly, labeled neurons located within the ipsilateral and contralateral lateral reticular nucleus (LRn) were plotted using a $20 \times$ objective and using a description of the nucleus provided by Ruigrok (2004) (also see Newman and Ginsberg, 1992). From these plots, usually consisting of 18-20 transverse levels (at $160 \mu \mathrm{m}$ interval), three-dimensional figurines of the left and right LRn were prepared with Neurolucida software. Counts of labeled neurons within the basal pontine nuclei (Pn) were constructed in a similar way (cases 864 and AP10). Additional pontine data were also obtained from Pijpers and Ruigrok (2006).

A Leica (Nussloch, Germany) DMR microscope equipped with a digital camera (Leica DC-300) was used to obtain photomicrographs. Photo panels were constructed in CorelDraw 11.0, after some correction for brightness and contrast in Corel Photopaint 11.0.

The individual cerebellar plots were used to construct the labeled mossy fiber terminals and climbing fiber collaterals in 2D reconstructions of the unfolded cerebellar surface (for a more detailed description, see Ruigrok, 2003). In short, the Purkinje cell layer, the underlying granular and overlying molecular layers were divided into $200-\mu \mathrm{m}$-wide mediolateral "bins." Within each bin, the number of labeled mossy fiber rosettes was charted and counted. Molecular layer bins were marked positive or negative for labeled climbing fiber terminals. Foliations and lobulations were followed throughout the transverse sections, and data were entered in serial order. In this way, a 2D matrix was constructed for each experiment (on average, each matrix was based on 40 transverse sections) that was visualized using MATLAB routines (The MathWorks, Natick, MA). This resulted in an interpolated and color-coded representation of the density of labeled mossy fiber rosettes over the entire unfolded cerebellar cortex. Note that these cerebellar cortical surface maps do not take into account the depth of the granular layer, which can vary considerably depending on the place and angle of sectioning. Hence, color coding of the mossy fiber rosettes does not reflect actual density concentrations, but rather reflects a general estimate of these densities. Because all cases were analyzed in the same way, the diagrams should still provide a useful means to assess and compare distributions of labeled mossy fiber rosettes. Localization of labeled climbing fiber collaterals was shown in a similarly constructed plot using MATLAB routines. Here, bins in which the molecular layer contained labeled climbing fiber terminals (regardless of their density) are indicated in red. In this way, a direct comparison was possible between the regional distribution of labeled mossy fibers and the presence of climbing fiber collaterals. Injection sites were indicated by a white box and arrow.

To further visualize how labeled mossy and climbing fibers were distributed over and into the depths of a folium, 3D reconstructions of the simple lobule (SL) were obtained in three cases using Neurolucida 3D reconstruction software and using a one of four series of sections. Section by section alignment was performed using the Neurolucida software. The midline ventral surface of the fourth ventricle was used as a reference and new sections were shifted and rotated to obtain a best fit of contours. Three-point smoothing of contours was performed before final 3D rendering by Neurolucida.

In two cases (AP03 and A41), 3D plots of mossy fiber and climbing fiber labeling within the simple lobule and vermal lobule VIa were constructed together with zebrin-positive Purkinje cells (also based on a one

\section{Table 2. Distribution (expressed as a percentage of the total number) of labeled mossy fiber rosettes in the granular layer subjacent to zebrin-positive and zebrin- negative bands in simple lobule and vermal lobule Vla}

\begin{tabular}{lcc}
\hline & C1 (AP03) & C2 (A41) \\
\hline Zebrin-positive band & & \\
P1+ & 0.0 & 2.3 \\
a + & 0.6 & 0.2 \\
P2 + & 0.1 & 5.3 \\
b+ & 0.0 & 3.8 \\
P4+ & 1.7 & 18.2 \\
P5 + & 3.2 & 22.2 \\
P6+ & 11.2 & 30.8 \\
Total (\%) & 16.7 & 82.7 \\
Fraction of granular layer subjacent to & 35.4 & 31.3 \\
$\quad$ zebrin-positive bands (\%) & & \\
Zebrin-negative band & & \\
P1- & 1.1 & 0.1 \\
a- & 2.1 & 0.0 \\
P2- & 0.9 & 0.9 \\
b- & 10.8 & 4.1 \\
P4- & 62.7 & 8.6 \\
P5- & 5.7 & 3.6 \\
Total (\%) & 83.3 & 68.7 \\
Fraction of granular layer subjacent to & 64.6 & \\
zebrin-negative bands (\%) & &
\end{tabular}

Data are shown for two cases: case AP03 with an injection into the $\mathrm{C} 1$ zone in PMD, and case A41 with an injection into the $\mathrm{C} 2$ zone in crus $2 \mathrm{~b}$ (see also Fig. 6). Note that a P3 + and a P6 - band are not clearly present in the simple lobule (Voogd and Ruigrok, 2004). 
in four series of sections). The plots of the individual sections were used to determine the proportion of the total number of labeled mossy fiber rosettes in the granular layer that were directly subjacent to zebrin-positive or zebrinnegative bands. Borders between zebrin bands in the molecular layer were extrapolated into the granular layer. To decide whether the distribution of labeled mossy fiber rosettes was biased toward a preferred position subjacent to either zebrin-positive or zebrin-negative bands, the surface areas of contours of the granular layer subjacent to these bands were determined and summed with Neurolucida. These data indicate that the total surface area of granular layer subjacent to zebrin-negative bands is approximately twice as large as that below zebrinpositive bands (Table 2).

In selected cases, the terminal labeling of mossy and climbing fibers was also combined with a standardized surface reconstruction in which the zebrin-banding pattern was shown (Pijpers et al., 2005). Zebrin bands labeled in the sections for each case were compared with the standardized reconstruction to accurately position the observed terminal labeling on the standard map. In the figures, roman numerals indicate individual lobules, and arrows are used to indicate their apices. The zebrin-positive bands are presented in gray, and the numbers and letters in these bands refer to the zebrinpositive compartments as defined by Hawkes and Leclerc (1987) and modified by Voogd and Ruigrok (2004) and by Sugihara and Shinoda (2004). The corresponding negative bands are positioned directly lateral to the positive bands. Because the labeling was indicated in a standardized diagram, the resulting diagrams may reflect some minor qualitative differences with the 2D diagrams (compare Figs. 2, 5).

\section{Results}

Distribution of climbing and mossy fiber collateral terminals: general observations

Iontophoretic CTb injections into the cerebellar cortex of the rat typically result in small, approximately spherical, deposits of tracer (diameter, $\sim 500 \mu \mathrm{m}$ ) (Ruigrok, 2003; Voogd et al., 2003). Because injections were placed at a depth of 200-300 $\mu \mathrm{m}$, they generally included the molecular layer and the underlying granular layer. The white matter was involved only to a very limited extent or not at all (Fig. 1A). Labeled parallel fibers and corresponding extensive retrograde labeling of granule cells usually obscured the boundaries of the injection site. As a result, mossy and climbing fiber labeling directly surrounding the injection site was interpreted with caution.

Retrograde cell labeling in the contralateral inferior olive was used to determine whether the site of injection was confined to a single cerebellar cortical zone and to identify that zone (Fig. $1 B$, inset, see arrow). Labeling confined mostly or wholly to the ventral fold of the dorsal accessory olive (vfDAO) was considered to be derived from the $\mathrm{C} 1$ zone (four cases), whereas the $\mathrm{C} 2$ zone (four cases) was targeted when the labeling was predominantly found in the rostral half of the medial accessory olive (MAO). In
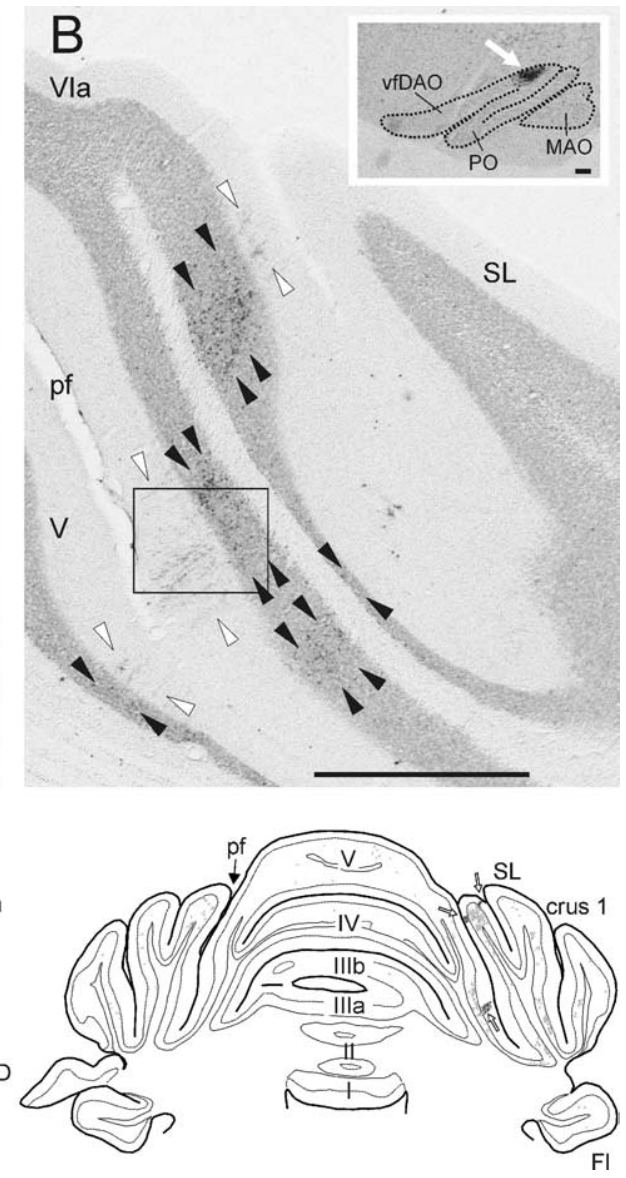

Figure 1. Photomicrographs showing aspects of the CTb injection site and resultant labeling of climbing and mossy fiber collaterals in case AP03 together with a selection of plots of transverse sections through cerebellum of case AP09. A, Photomicromolecular and granular layers of the electrophysiologically identified C1 zone, which was verified by the presence of retrograde labeled cells in the contralateral vfDA0 ( $\boldsymbol{B}$, inset). $\boldsymbol{B}$, Photomicrograph showing part of a section of the SL and anterior lobe of the and the numerous labeled mossy fiber rosettes within the granular layer (between black arrowheads). Also note that the regions dhe mediolateral plane but that the regions with mossy fiber terminal rosettes are also contralateralinferior olive. Magnification of the boxed rectangle in B showing the boundary of granular and molecularlayer. The arrowheads indicate some of the labeled mossy fiber rosettes that are located directly adjacent to labeled climbing fiber terminal arborizations. $\boldsymbol{D}$, Two plots of transverse sections through the posterior and anterior cerebellum, respectively, and indicating the distribution of (Tb labeling resulting from a PMD injection in the electrophysiologically identified (2 zone (case AP09). Labeled mossy fiber rosettes (small gray dots: one dot equals one rosette) and climbing fiber terminals (black dots indicated by arrows) are indicated. Scale bars: $\boldsymbol{A}, \boldsymbol{B}, 1 \mathrm{~mm} ; \boldsymbol{B}$, inset, $100 \mu \mathrm{m} ; \boldsymbol{C}, 100 \mu \mathrm{m}$. I-X, Lobules I-X; FI, flocculus; pf, primary fissure.

three additional cases, most labeled neurons were located in the ventral leaf of the principal olive (PO) and/or the dorsomedial group (DM) of the $\mathrm{PO}$, indicating that the injection site mostly covered the D1 and/or D0 zones, respectively (Table 1). Retrograde cell labeling was also noted in several brainstem nuclei known to provide mossy fibers to cerebellar lobules VII and VIII, such as the LRn, Pn, and, more sparsely, the dorsal column nuclei, trigeminal nucleus, and reticular formation.

Because $\mathrm{CTb}$ is a bidirectional tracer, it was also transported to the terminal arborizations of collateral branches of the stem axons of retrogradely labeled cells, so-called collateral-collateral labeling (Chen and Aston-Jones, 1998; Ruigrok, 2003; Voogd et al., 2003). Labeled terminal rosettes of mossy fiber collaterals generally displayed a more extensive distribution over the cerebellar cortex than labeled climbing fiber collaterals (Fig. $1 B, D$ ). Apart from sending collaterals ipsilateral to the injection site, 

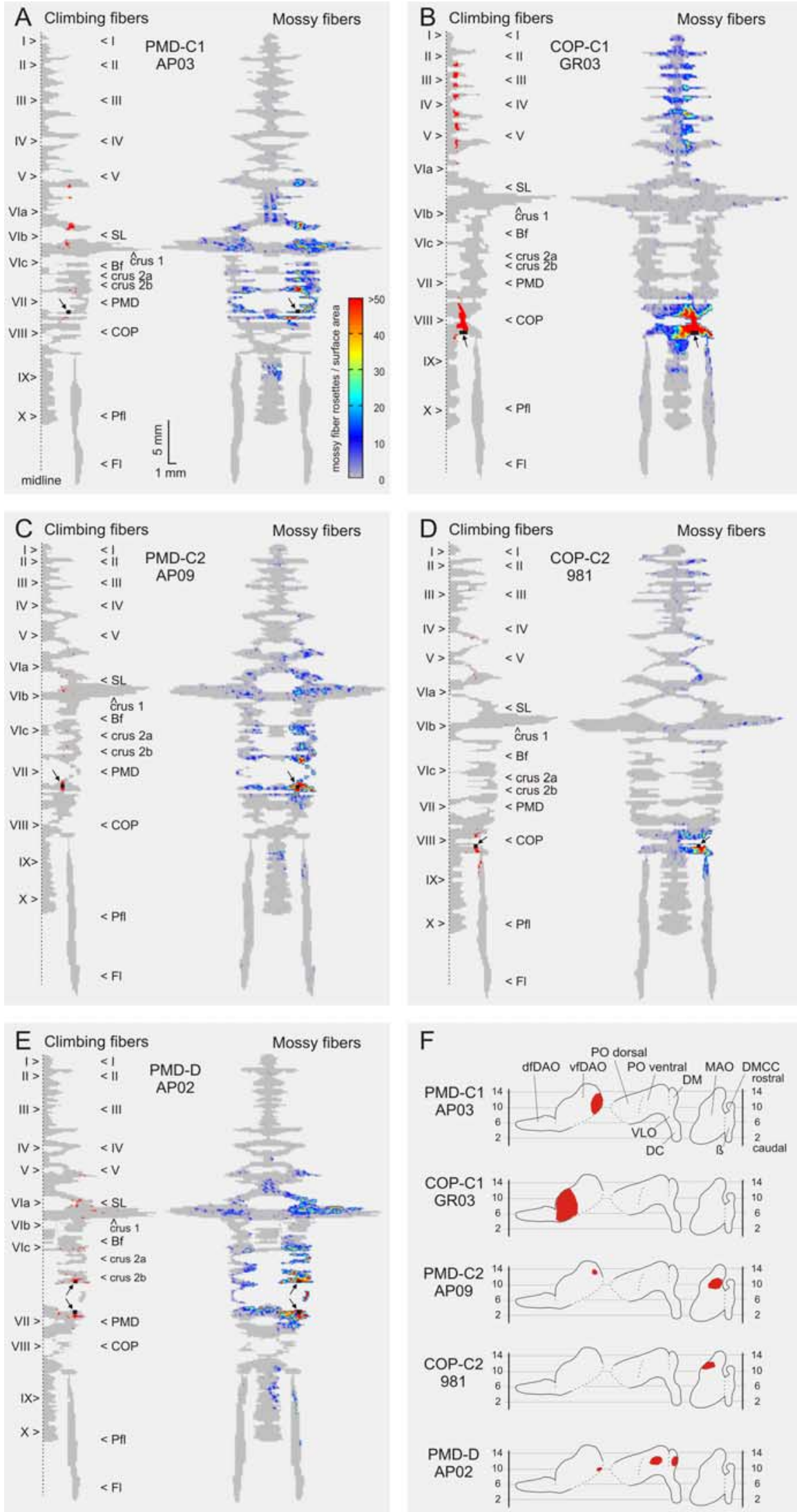

Figure 2. Color diagrams summarizing the results of five cases with injections in the $\mathrm{C} 1$ zone in PMD (case AP03) ( $A),(1$ zone in COP (case GR03) (B), C2 in PMD (case AP09) (C), C2 in COP (case 981) (D), and the D zone in PMD (case AP02) (E), as well as generalized flattened surface diagrams indicating the position of resultant cell labeling (red) in the inferior olivary complex $(\boldsymbol{F})$. The left-hand panels show a representation of the right one-half of the unfolded and flattened surface diagram of the cerebellar cortex. The arrows and their roman numeral indicate the apex of vermal and hemispheral lobules. The injection sites are indicated labeled mossy fiber terminals were also usually found contralaterally, arranged in an approximately symmetrical pattern to the distribution on the ipsilateral side, although the density was generally lower. Terminal arborizations of labeled climbing fiber collaterals were confined ipsilaterally within the molecular layer of both the anterior and posterior cerebellum, usually in rostrocaudally oriented strips that were usually a few and up to $\sim 10$ Purkinje cells wide (Fig. $1 B-D$ ).

Figure $1 D$ shows examples of transverse plots from which the 2D cortical surface (Fig. 2) as well as the $3 \mathrm{D}$ reconstructions (Figs. 3, 6) were derived. The distribution of all labeled mossy fiber rosettes in the granular layer are shown as gray dots, whereas the general location of labeled climbing fiber collaterals in the molecular layer are shown in black (open arrows).

\section{Distribution of climbing and mossy fiber collateral terminals: the $\mathrm{C} 1$ zone}

Figure 2 illustrates five cases with injections into the $\mathrm{C} 1$ zone (Fig. $2 A, B$ ), the $\mathrm{C} 2$ zone (Fig. $2 C, D$ ), and the $\mathrm{D}$ zone (Fig. $2 E$ ) plotted onto $2 \mathrm{D}$ surface maps. In case AP03 (Fig. 2A), the CTb injection involved the $\mathrm{C} 1$ zone of the $\mathrm{PMD}$, whereas $\mathrm{C} 1$ of the COP was injected in case GR03 (Fig. 2B). The injection in case AP03 was guided by previous recording of the distribution of climbing fiber field potentials on the surface of the cerebellum, evoked by ipsilateral forelimb stimulation. These responses identify the mediolateral boundaries of the $\mathrm{C} 1$ zone in PMD (cf. Atkins and Apps, 1997). The exclusive C1 zone location of this injection site was confirmed by the presence of retrogradely labeled neurons confined to the medial aspect of vfDAO (Fig. 2F, top panel, flattened surface reconstruction of olivary subnuclei). Figure 2A, left-hand panel, shows the position of labeled climbing fibers on an unfolded surface map of the

\footnotetext{
$\leftarrow$

by a black square and arrow. The red labeling indicates the presence of bins with labeled climbing fiber terminals in the molecular layer. The right-hand panels show a similar diagram of the whole cerebellar cortex, but now representing the granular layer. Color coding indicates the number of labeled mossy fiber rosettes in 200- $\mu \mathrm{m}$-wide bins of the granular layer (see color bar in $A$ ). For additional details on cortical reconstruction, see Ruigrok (2003) and Materials and Methods, Analysis. For a detailed description of individual cases, see Results. I-X, Lobules $I-X ; \beta, \beta$ subnucleus; Bf, buried folium; $D C$, dorsal cap; $\mathrm{dfDAO}$, dorsal fold of dorsal accessory olive; DMCC, dorsomedial cell column; Fl, flocculus; Pfl, paraflocculus; VLO, ventrolateral outgrowth.
} 
cerebellar cortex (Ruigrok, 2003; Pijpers et al., 2005). From this diagram, it is evident that the climbing fiber collaterals were distributed to the paravermal cerebellum as continuous strips or, occasionally, as isolated patches that fell within a longitudinal band of cortex that was aligned with the injection site (Fig. $2 \mathrm{~A}$, shown in black, arrow). More specifically, from the injection site caudalward, labeled climbing fibers were noted in the caudal one-half of PMD (lobule VII) and rostralward in crus $2 \mathrm{~b}$, crus 1a, SL, and caudal one-half of lobule $\mathrm{V}$. In Figure $2 \mathrm{~A}$, right-hand panel, the distribution of labeled mossy fiber rosettes is depicted on the same 2D surface map in color-coded density profiles (Ruigrok, 2003). In the ipsilateral one-half of the cerebellar cortex, the overall distribution pattern of the mossy fiber terminals shows accumulations within three mediolateral regions. Isolated patches of labeled mossy fibers were present within the vermis of lobules VI and VII and IX and, as a more or less continuous strip, in the hemisphere of PMD and crus 2, but were also noted in SL and crus 1 . Most terminals, however, were located within the paravermal cerebellum, distributed mainly within lobules V-rostral VIII. The pattern of vermal and paravermal mossy fiber terminal labeling is approximately mirrored on the contralateral side at a lower density. However, contralateral labeling was not observed in lobules V and IX or in the lateral-most part of the hemisphere. When comparing the distributions of climbing fiber and mossy fiber terminal labeling, it is also apparent that at all sites where climbing fiber collaterals are situated, a high density of labeled mossy fiber rosettes is also usually present in the subjacent granular layer (Fig. $2 \mathrm{~A}$, compare the location of labeling in the left- and right-hand maps).

In case GR03 (Fig. 2B), the injection, which was based on anatomical coordinates, was made into the hindlimb-related part of the C1 zone in COP (Atkins and Apps, 1997). Retrogradely labeled neurons in the contralateral inferior olive were confined to the lateral part of vfDAO (Fig. $2 F$, second panel), confirming that the injection site was restricted to the $\mathrm{C} 1$ zone. Although some labeled climbing fibers entered the dorsolateral aspect of lobule IX, most labeled climbing fiber collaterals emerged from the injection site toward the rostral part of lobule VIII. In the anterior lobe, a conspicuous and continuous rostrocaudally oriented strip of labeled climbing fibers was present in the paravermal cortex stretching from lobules V to II (Figs. $2 B$, left-hand panel; compare Fig. 5). Labeled mossy fiber rosettes (Fig. $2 B$, right-hand panel) were abundantly present throughout the mediolateral extent of lobule VIII ipsilaterally, whereas they were confined to vermal and paravermal parts contralaterally. In addition, patches of labeled rosettes were observed in isolated

White matter contour
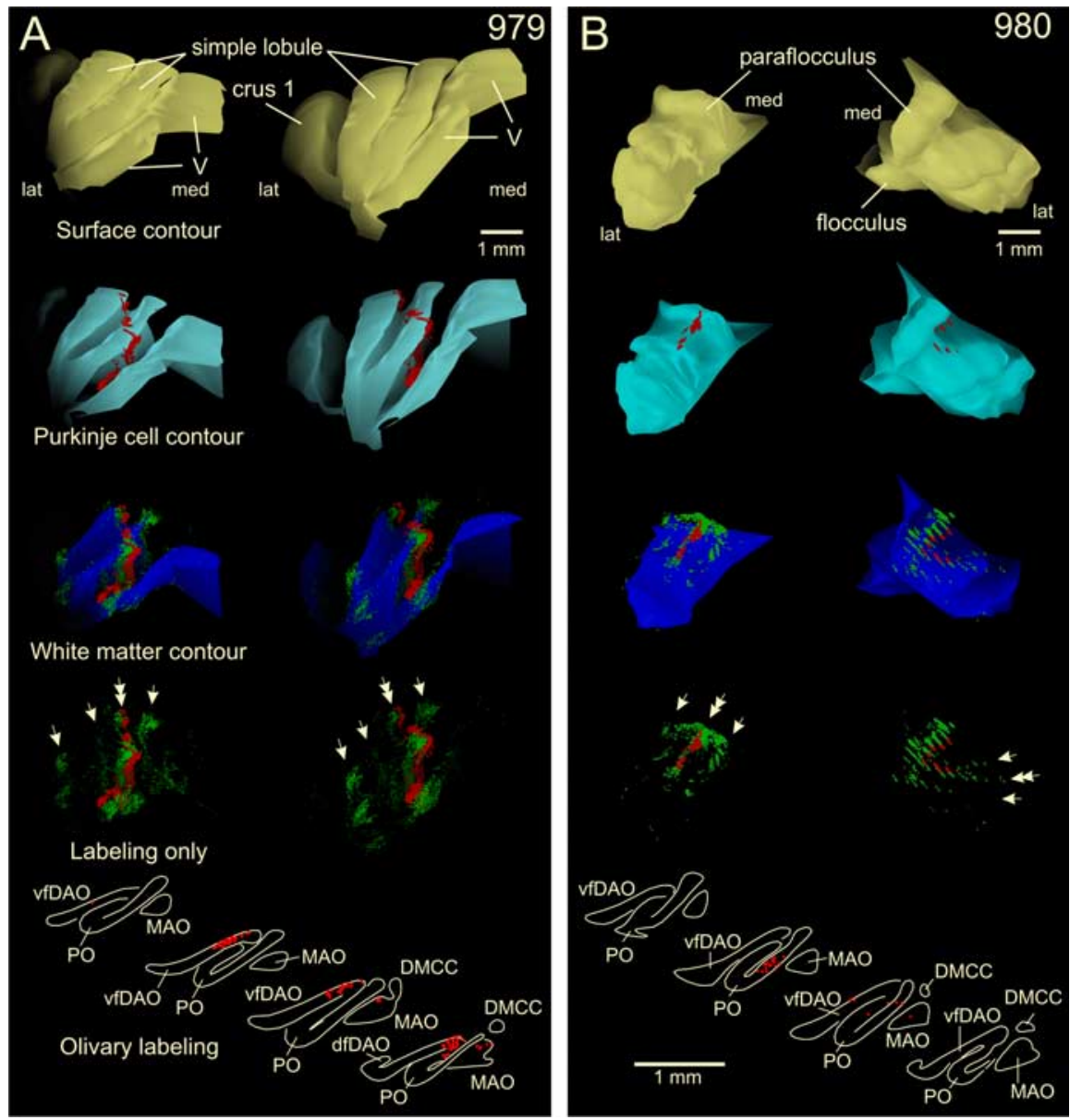

Figure 3. 3D reconstructions of part of the cerebellar cortex showing the correspondence of mossy and climbing fiber collateral labeling. $A$, Diagrams representing case 979 with a CTb injection centered on $C 1$ of PMD, with some involvement of $C 2$ as judged by the distribution of retrogradely labeled neurons in the inferior olive (bottom panels). $3 \mathrm{D}$ reconstructions show a rostromedial (left-hand column of panels) and a rostral (right-hand column of panels) view of the ipsilateral simple lobule and adjacent parts of lobule $V$ and crus 1 . From the top down, the pial surface contour is shown in yellow, the contour indicating the Purkinje cell monolayer in cyan, with the labeled climbing fiber terminal labeling superimposed in red. Subsequently, the white matter contour panels indicate labeled mossy fiber rosettes and climbing fiber terminals only. Note that a strip of labeled mossy fiber rosettes a rostral dorsolateral view. In the latter view, the discontinuity of labeling is caused by the intermittent, nonplotted, sections (for labeled mossy fiber rosettes were noted (arrows), the middle of which was accompanied by labeled climbing fibers (double arrow). V, Lobule V; dfDA0, dorsal fold of dorsal accessory olive; DMCC, dorsomedial cell column; VLO, ventrolateral outgrowth.

vermal, paravermal, and hemispheral regions of lobules II-V ipsilaterally, and in mirror-like image in vermal and paravermal regions of the contralateral one-half of the anterior lobe. In contrast to the injection into the $\mathrm{C} 1$ zone in $\mathrm{PMD}$, virtually no labeled rosettes were encountered in lobules VI and VII, including the hemispheral regions of SL, crus 1, crus 2, and PMD. Again, the correspondence between the distribution of climbing fiber collaterals and mossy fiber collaterals is striking.

Distribution of climbing and mossy fiber collateral terminals: the $\mathrm{C} 2$ zone

Figure 2, C (case AP09) and D (case 981), illustrates two examples of $\mathrm{CTb}$ injections that were centered on the $\mathrm{C} 2$ zone in the PMD and in the COP, respectively (Table 1). The C2 location of both 
A
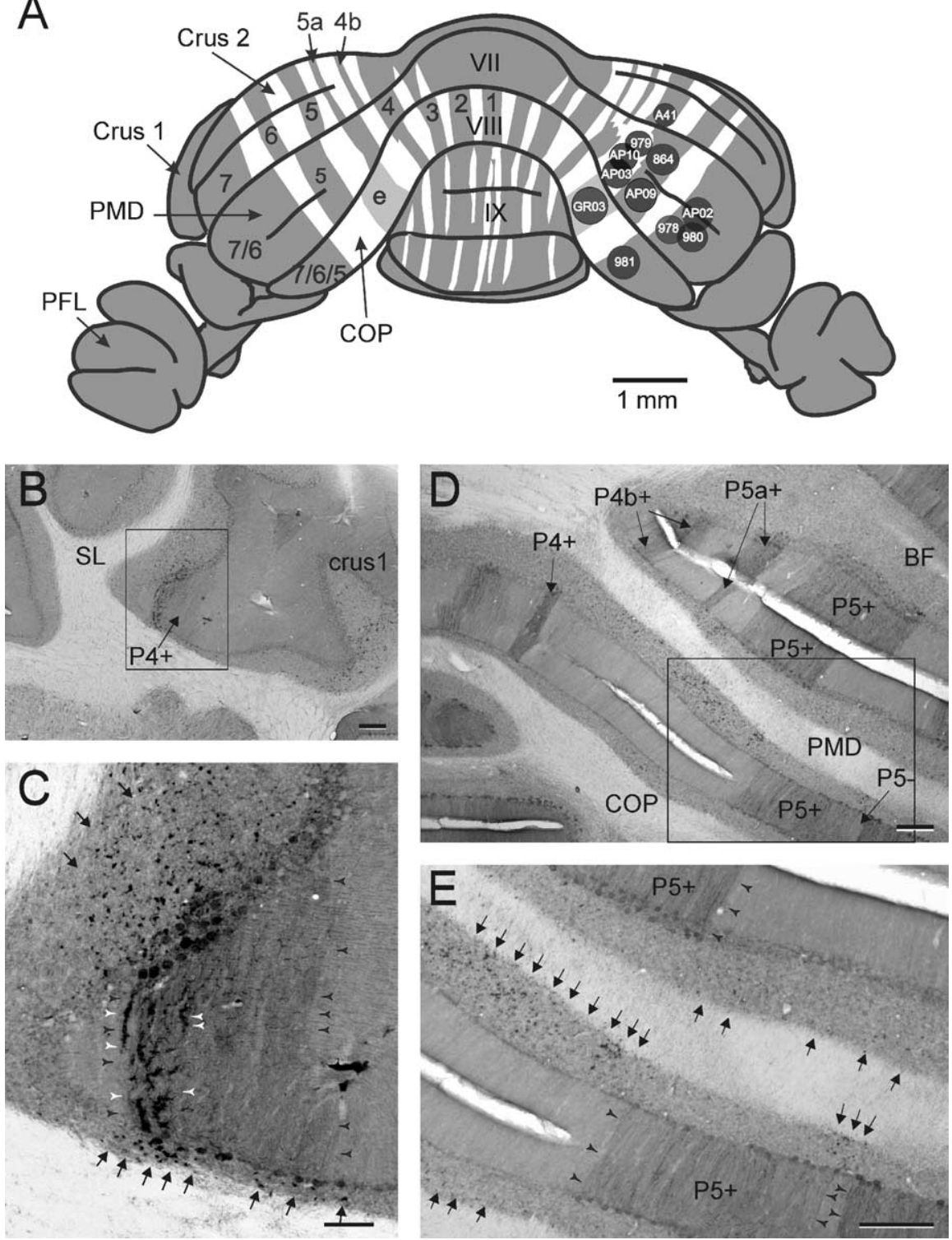

Figure 4. Relationship of injection sites and collateral labeling of climbing and mossy fibers with the intrinsic zebrin pattern of Purkinje cells. $\boldsymbol{A}$, Diagram showing the approximate site and size of the (Tb injections used in this study on a reconstruction of the posterior view of the rat cerebellum stained for zebrin (Voogd et al., 2003; Pijpers and Ruigrok, 2006). Note that only the positive zebrin bands are indicated by their respective numbers and letters. Patch " $\mathrm{e}$ " is shaded light gray in accordance with the lightly zebrin-positive nature of this patch. $\boldsymbol{B}$, Photomicrograph taken from case A41, depicting zebrin-positive band P4+ (arrow) in SL. $\boldsymbol{C}$, Detail of boxed area in $\boldsymbol{B}$. Note that darkly stained climbing fiber collaterals (white arrowheads) are found over the medial part of $\mathrm{P} 4+$ (width of band indicated by black arrowheads). The arrows point to labeled mossy fiber rosettes, which are found subjacent to $\mathrm{P} 4+. \boldsymbol{D}, \boldsymbol{E}$, Photomicrographs taken from case AP03 showing the relationship of mossy fiber rosettes with negative zebrin bands in the posterior cerebellum. Zebrin-positive bands and band P5 - are labeled ( $\boldsymbol{D}$, arrows). Note that, in $\boldsymbol{E}$ (showing the boxed area of $\boldsymbol{D}$ in higher magnification), labeled rosettes (arrows) are present subjacent to several zebrin-negative areas. Also note that no climbing fiber collaterals were labeled in the zebrin-negative bands. Scale bars: $\boldsymbol{A}, 1 \mathrm{~mm} ; \boldsymbol{C}, 100 \mu \mathrm{m} ; \boldsymbol{B}, \boldsymbol{D}, \boldsymbol{E}, 250 \mu \mathrm{m}$. VII, VIII, IX, Cerebellar lobules VII, VIII, IX; BF, buried folium; PFL, paraflocculus.

injection sites was confirmed by the fact that most of the retrogradely labeled neurons in the inferior olive were observed in the rostral part of the MAO (Fig. $2 F$, third and fourth panels). The few additionally labeled neurons in the medial part of vfDAO in case AP09 suggest, however, some involvement of the neighboring $\mathrm{C} 1$ or $\mathrm{C} 3$ zone. The distribution of labeled climbing fiber collaterals in this case was found within several narrow and isolated regions of the paravermis within crus 2, SL, and lobule V, which, in general were well aligned along the rostrocaudal axis with the injection site (Fig. 2C, left-hand panel). Labeled mossy fiber rosettes (Fig. 2C, right-hand panel) were mostly present in the paravermal regions of the same lobules (i.e., lobules V, VI, and VII). However, patches of labeled terminals were also found in the hemispheral region of SL, both crura, lobule VIII and in the caudal parts of the proximal paraflocculus. Some vermal patches were noted in lobules V-VII and IXa. Contralateral labeling was mostly confined to paravermal regions. Note again that all places with climbing fiber terminal labeling also contained labeled mossy fiber rosettes in the subjacent granular layer.

The same observation was made for case 981 in which the very small injection was centered on the $\mathrm{C} 2$ zone in the COP (Fig. 2D). However, here, areas of overlap of labeled climbing and labeled mossy fibers was found in paravermal regions of lobules III-V and in lobule VIII and proximal part of the paraflocculus. Isolated patches without corresponding climbing fiber labeling were also noted ipsilaterally in lobule II, lateral crus 1, and in vermal and lateral parts of the rostral one-half of lobule VIII. In this case, virtually no labeled mossy fiber rosettes were observed on the contralateral side.

\section{Distribution of climbing and mossy} fiber collateral terminals: D0-D1 zones Figure $2 E$ shows the results from an injection that aimed to target the $\mathrm{C} 3$ zone in PMD, but because most retrogradely labeled neurons in the olive were located in the DM group and the ventral leaf of the PO (Fig. $2 F$, bottom panel), it is likely that the injection deviated laterally to involve the D1 and D0 zones, respectively (Voogd et al., 2003). A few neurons in the medial DAO suggest, however, some incorporation of the $\mathrm{C} 3$ zone. The distribution of labeled climbing fiber collaterals was rather dispersed mediolaterally, involving paravermal and hemispheral locations, presumably because of the involvement of multiple zones (Fig. 2E, left-hand panel). Similarly, labeled mossy fibers were also widespread and were located in vermal, paravermal, and hemispheral regions of lobules V-VII. Note, however, that the hemispheral component did not have a bilateral counterpart (Fig. $2 \mathrm{E}$, right-hand panel). Importantly, and despite the scattered distribution of climbing fiber collaterals, they were always colocalized with labeled mossy fiber rosettes.

\section{Distribution of climbing and mossy fiber collateral terminals: $3 \mathrm{D}$ reconstructions}

The spatial distribution of climbing and mossy fiber collateral terminals relative to one another can be more directly appreciated in a $3 \mathrm{D}$ representation. Figure $3 A$ shows a $3 \mathrm{D}$ reconstruction of lobule V, SL, and the anterior part of crus 1 for case 979 (see 
also Fig. 5). In this experiment, the injection site was mostly confined to the $\mathrm{C} 1$ zone in PMD (Fig. 4A), as was confirmed by the majority of labeled olive neurons being located in the medial aspect of vfDAO (Fig. 4A, bottom panel; Table 1). Cerebellar surface contours (yellow), Purkinje cell layer (cyan), and white matter contours (dark blue) indicate foliations together with the location of labeled climbing fiber collaterals (red) in the molecular layer and individual labeled mossy fiber rosettes (green). In this example, a single more or less continuous, rostrocaudally oriented, strip of collateral climbing fiber labeling could be followed from the caudal bank of lobule $\mathrm{V}$ into the depths of the primary fissure and then into lobules a and $\mathrm{b}$ of SL. Along this entire route, the location of labeled climbing fibers is closely tracked by labeled mossy fiber rosettes (double arrow), which, in addition, also form isolated strip-like clusters medial and lateral to the labeled climbing fibers (arrows). Note that, although the strip of labeled mossy fibers is almost continuous throughout the depths of the primary fissure, the patches of mossy fiber rosettes at the lateral border of SL are not continuous and are found only at the apex of the two SL folia.

Figure $3 B$ shows a $3 \mathrm{D}$ reconstruction for a different case (case 980), depicting labeling of climbing and mossy fiber collateral terminations in the paraflocculus. Retrograde cell labeling of olivary neurons in the ventral leaf of PO indicated that the injection site was located in the $\mathrm{D} 1$ zone in PMD (Fig. 4A). Three strip-like accumulations of labeled mossy fibers are present, which, in accordance with the specific foliation of the paraflocculus, run essentially in a mediolateral direction. The middle of these three strips is precisely accompanied by labeled climbing fiber collaterals.

\section{Distribution of climbing and mossy fiber collateral terminals: relationship to the zebrin pattern}

To further describe how the non-climbing fiber-related mossy collaterals were distributed over the cerebellar cortex, labeling was also analyzed in sections that were stained for zebrin II immunohistochemistry. In Figure 4, the injection sites of all 11 cases are shown on the right side of a generalized caudal view of the cerebellar cortex in which the zebrin pattern has also been indicated. Note that, in each case, the injection site was located in either a zebrin-positive or a zebrin-negative band. For example, in case A41, the injection site was considered to be within the confines of P5+ in crus $2 \mathrm{~b}$. Photomicrographs in Figure $4 B$ and, in detail, in $C$, illustrate how the resultant collateral accessory olive.

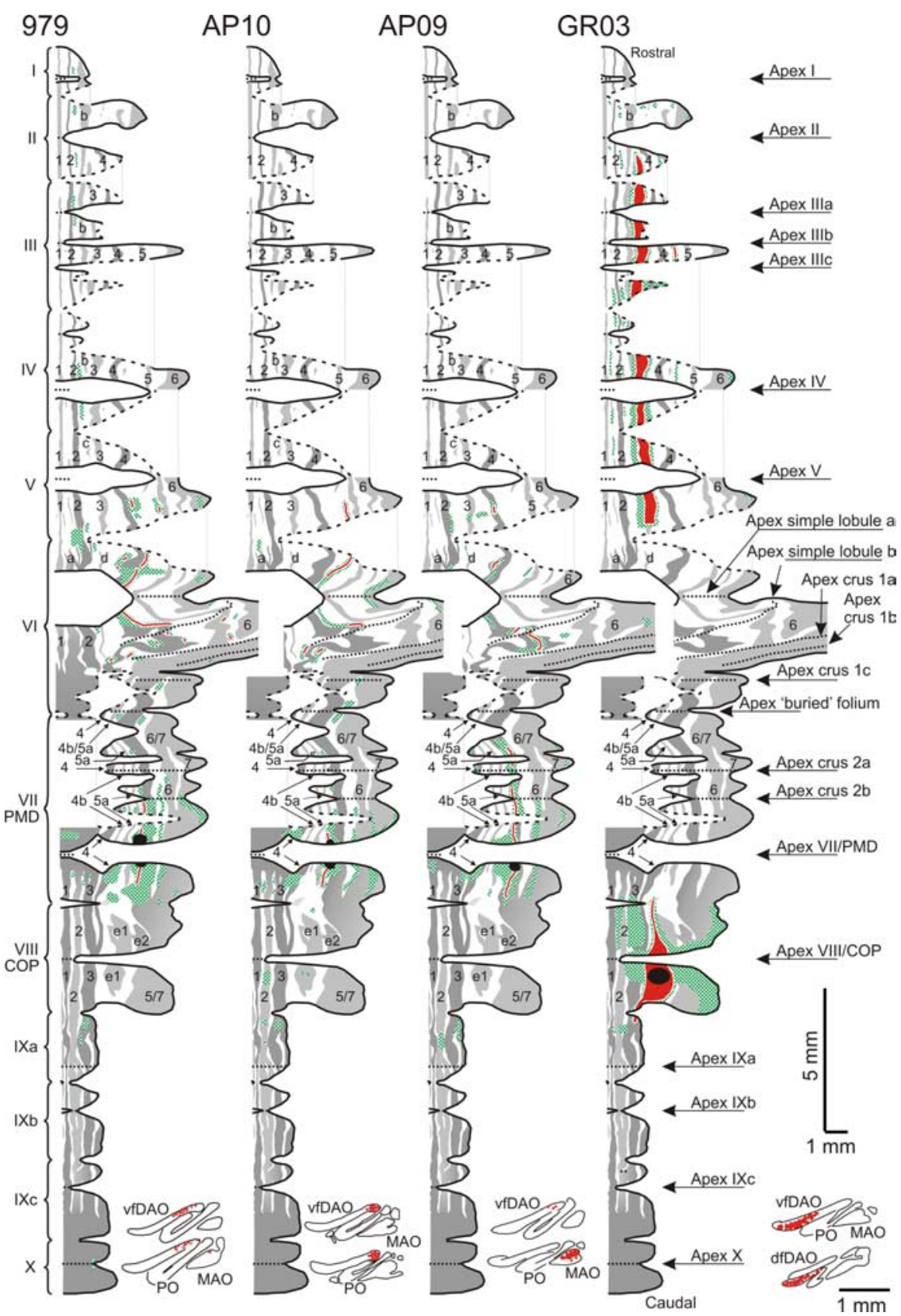

Figure 5. Distribution of labeled mossy fiber rosettes and labeled climbing fiber collaterals of four cases plotted on a standardized surface reconstruction of the zebrin pattern of Purkinje cells (Pijpers et al., 2005). Roman numerals indicate individual lobules and arrows are used to indicate their apices. The zebrin-positive bands and their continuity over the cerebellar cortex is presented in shades of gray, and the numbers and letters in these bands refer to the zebrin-positive bands from the nomenclature of Hawkes and Leclerc (1987) and modified by Voogd and Ruigrok (2004) and by Sugihara and Shinoda (2004). The corresponding negative bands lie immediately lateral to these positive bands. Labeling of both mossy and climbing fiber terminals was qualitatively entered on the standardized diagram by examining sections double labeled for zebrin and $\mathrm{CTb}$ and using the zebrin pattern as a reference frame. Regions in the granular layer with clustered presence of labeled mossy fiber rosettes are indicated with hatched patterns in green; climbing fiber terminal labeling is indicated in red in the same diagrams. The figurines at the bottom of each diagram show the localization of retrogradely labeled neurons in representative sections through the inferior olivary complex. For explanation of the individual diagrams, see Results, Distribution of climbing and mossy fiber collateral terminals: relationship to the zebrin pattern. $1-7$, Zebrin-positive bands $\mathrm{P} 1+$ to $\mathrm{P} 7+; \mathrm{I}-\mathrm{X}$, lobules $\mathrm{I-X}$; Bf, buried folium; dfDA0, dorsal fold of dorsal

labeling of climbing fiber terminations $(C$, white arrowheads) correlate with the zebrin-positive band $\mathrm{P} 4+$ in the caudal part of SL and that labeled mossy fiber rosettes are also confined to regions of the granule cell layer below the $\mathrm{P} 4+$ band $(C$, black arrows). In comparison, the photomicrographs in Figure $4 D$, 

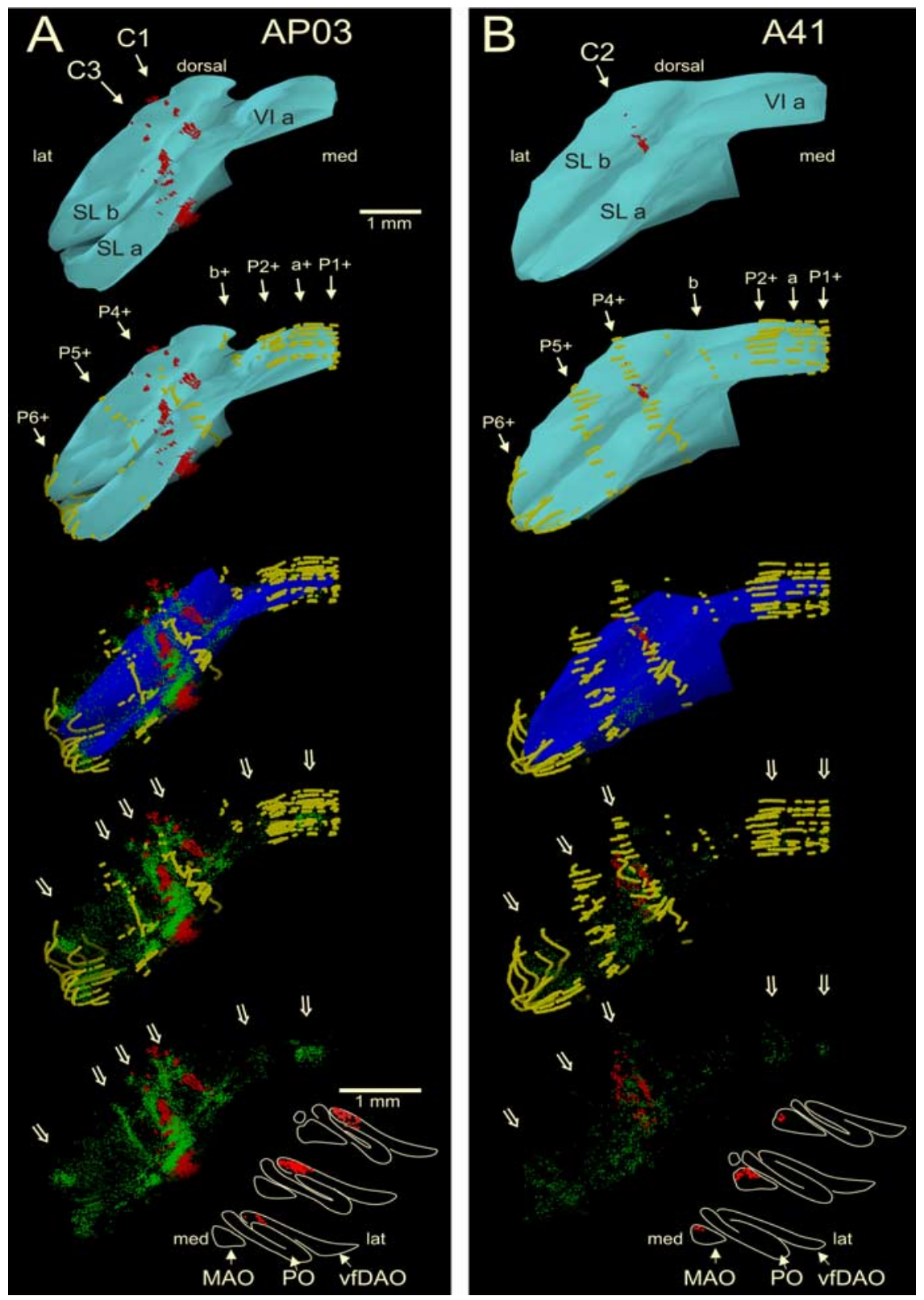

Figure 6. Rostral view of three-dimensional color reconstructions of part of the simple lobule of cases AP03 $(\boldsymbol{A})$ and A41 (B) showing the correspondence of mossy and climbing fiber collateral labeling in relation to the pattern of zebrin-positive Purkinje cells (shown in brown; other conventions are similar to those of Fig. 3). $A$, In case AP03, the injection site was confined to the $C 1$ zone of PMD as was evident by labeling of olivary neurons in the medial part of the vfDAO (bottom of panel). In SL, labeled climbing fiber collaterals are found in the P3 - and P4 - bands relating to the 11 and 3 zones (Pijpers et al., 2005). Labeled mossy fibers rosettes are found subjacent to these labeled climbing fibers and, in addition, are also found at other and nonadjacent locations (open arrows). Note that most mossy fiber labeling is positioned subjacent to zebrin-negative areas. $\boldsymbol{B}$, In case A41, the injection site was centered on the $\mathrm{C} 2$ zone of crus $2 \mathrm{~b}$ and resulted in labeled olivary neurons in the rostral MAO. In SL, labeled climbing fiber collaterals are found within the P4 + band that relates to the (2 zone (Pijpers et al., 2005). Labeled mossy fibers rosettes are found subjacent to these labeled climbing fibers and, in addition, are also found at other nonadjacent locations (open arrows). Note that many of them are related to zebrin-positive areas. P1 + to P6 + a, and b refer to zebrin bands (Voogd and Ruigrok, 2004). VI a, Lobule VI a; SL a, b, simple lobule a, b. Olivary panels are arranged from rostral (bottom) to caudal (top).

and an enlargement shown in Figure $4 E$, are taken from case AP10, which was centered on the zebrin-negative band P5a- in PMD. Mossy fiber rosettes (black arrows) can be seen to be mostly located subjacent to zebrin-negative areas. Note that, in Figure $4, D$ and $E$, no labeled climbing fiber collaterals were present in the molecular layer.

Figure 5 details for four representative cases the relationship between the distribution of labeled mossy and climbing fiber collaterals (hatched green and solid red, respectively), and the pattern of zebrinpositive and -negative Purkinje cells as shown in a generalized cortical surface map (for details, see Pijpers et al., 2005). A black circle in each diagram represents the approximate location of the injection site in each case. Parts of the diagram representing the flocculus, paraflocculus, and the lateral part of crus 1 are not shown, because these regions are fully zebrinpositive and the illustrated cases only displayed very limited mossy fiber collateral labeling in these areas.

From these diagrams, it can be appreciated that $\mathrm{C} 1$ zone injection sites (cases 979 and AP10) resulted in accumulations of labeled mossy fiber rosettes predominantly below zebrin-negative Purkinje cells, whereas in cases with $\mathrm{C} 2$ zone injection sites (e.g., case AP09) they are mostly found in relation to zebrin-positive bands. The injection site in case GR03 was determined to be within the $\mathrm{C} 1$ zone in $\mathrm{COP}$; however, it appeared to be centered on the rather weakly zebrin positive patch $\mathrm{e} 1+$. Climbing fiber collaterals were mostly distributed to the also weakly zebrin-positive band $\mathrm{P} 3+$ in the anterior lobe. Both e1+ and $\mathrm{P} 3+$ and their bordering zebrinnegative bands have been shown to be located fully within the $\mathrm{C} 1$ zone (Voogd et al., 2003; Sugihara and Shinoda, 2004). Also, most additional mossy fiber patches in the anterior lobe were related to zebrinnegative bands. For these reasons, both zebrin patch $\mathrm{e} 1+$ as well as anterior lobe band P3 + may be considered to be constituting a specific part of zebrin-negative territory. Note that, regardless of the location of the injection site in zebrin-negative or -positive bands, all cases demonstrated at least some patches of mossy fibers within the lateral-most zebrin-positive band.

The relationship between the intrinsic zebrin pattern of Purkinje cells and the distribution of climbing and mossy fiber collaterals is further illustrated in Figure 6, which shows $3 \mathrm{D}$ reconstructions of the $\mathrm{SL}$ for two cases. Case AP03 (Fig. 6A) had an injection that was centered on the $\mathrm{C} 1$ zone in PMD (also shown in Fig. 2A), whereas the injection in case A41 (Fig. 6B) involved the $\mathrm{C} 2$ zone in crus $2 \mathrm{~b}$. In the top two panels in both Figure 6, $A$ and $B$, the Purkinje cell layer/granule cell layer interface is shown in cyan together with the labeled climbing fiber collaterals in the molecular layer (red). Zebrin-positive Purkinje cells (yellow) have been added in the second pair of panels. In the third pair of panels, the location of labeled mossy fiber rosettes have been added (green) and all three classes of labeling are shown in relation to the white matter contours (dark blue) to indicate foliations. In the bottom two cerebellar representations, 
only the labeled climbing and mossy fiber terminals are shown either with or without zebrin-positive Purkinje cells, respectively. Finally, the retrograde cell labeling within the inferior olive is shown to indicate the zonal localization of the injection site. Note that, in case AP03, strips of labeled climbing fiber collaterals are found in zebrin-negative regions on either side of the $\mathrm{P} 4+$ band, representing the $\mathrm{C} 1$ and $\mathrm{C} 3$ zones (Voogd et al., 2003). In contrast, in case A41, labeled climbing fiber collaterals were found only in the $\mathrm{P} 4+$ band, which represents the C2 zone (Voogd et al., 2003). In both cases, labeled mossy fiber collaterals were most abundant directly subjacent to the labeled climbing fiber collaterals. However, collections of labeled mossy fiber rosettes were found in other regions as well. Although not exclusively (note labeled mossy fibers within P6+ in both cases), these mossy fibers were mostly restricted to zebrin-negative bands in case AP03 and to zebrin-positive bands in case A41.

This finding was supported by quantitative analysis. Table 2 shows for the two representative cases the proportion of labeled mossy fiber rosettes in relation to zebrin-positive and -negative bands (for further details of the analysis, see Materials and Methods). From inspection of Table 2, it is evident that, subjacent to all zebrinpositive bands (apart from satellite band $\mathrm{a}+$ ), the percentage of labeled rosettes was always greater in case A41 (C2 injection) than in case AP03 (C1 injection), and that this situation was essentially reversed for mossy fiber labeling subjacent to zebrinnegative bands. It is also noteworthy that, in the $\mathrm{C} 1$ zone case, the only zebrinpositive band with a fair amount (11.2\%) of labeled rosettes in the subjacent granular layer was P6+. Distributions of mossy fiber collaterals to $\mathrm{P} 6+$ were also noted in other cases with injections into the $\mathrm{Cl}$ zone (Fig. 5), highlighting the atypical status of this particular hemispheral zebrin band.

In summary, mossy fiber and climbing fiber collaterals labeled after injections centered on the $\mathrm{C} 1$ zone in $\mathrm{COP}$ or PMD are mostly found in zebrin-negative bands (with the inclusion of the weakly zebrin-positive P3 + band in the anterior lobe). In contrast, mossy fiber and climbing fiber collaterals labeled after injections centered on the $\mathrm{C} 2$ zone are mostly located in zebrinpositive bands.

\section{Origin of mossy fibers to different lobules and zones}

The results above indicate that the distribution pattern of mossy fiber rosettes associated with the $\mathrm{C} 1$ zone differs from that of the C2 zone. However, lobular differences may also be present. To examine the possibility of zonal and lobular differences more fully, the distribution of retrograde cell labeling in two major sources of mossy fibers was studied: the Pn [based mainly on data recently published by Pijpers and Ruigrok (2006)] and also the LRn.

In Figure 7, the distributions of retrogradely labeled cells in three-dimensional views of both the ipsilateral and contralateral LRn are shown for all cases studied. Cases are presented with respect to the location of the injection site in the $\mathrm{C} 1$ zone in $\mathrm{PMD}$ or COP, the $\mathrm{C} 2$ zone in crus 2, PMD, or COP, and the D0/D1 zones in PMD. For each case, a predominantly caudal (at $10^{\circ}$ dorsal from the horizontal axis) as well as a predominantly dorsal view (at $70^{\circ}$ dorsal from the horizontal axis) is shown, in which similarities and differences in distribution of labeled neurons between cases can be easily appreciated.

For the C1-PMD and C2-PMD cases, labeled cells were distributed throughout the rostrocaudal extent of LRn, taking up mostly dorsal positions in its magnocellular and subtrigeminal 
parts, whereas many labeled neurons were also observed within its linear subdivision [for definition of subdivisions, see Newman and Ginsberg (1992); Ruigrok (2004)]. Most labeled neurons were observed ipsilaterally (Table 1), but labeled cells were also located at similar positions in the contralateral LRn (except the caudal aspect of the nucleus, which was virtually devoid of labeled neurons). Apart from a somewhat more pronounced involvement of the caudal one-third of the LRn in the $\mathrm{C} 1$ zone cases, no clear differences were observed between $\mathrm{C} 1$ and $\mathrm{C} 2$ injections in PMD. In comparison, in the D0/D1 cases, the main magnocellular and parvicellular parts of the LRn contained only a few labeled cells. Most labeled cells were found in the subtrigeminal and linear subdivisions. The distribution of labeled neurons in case A41 (C2 zone in crus 2b) was very similar to that of the three D0/D1 cases.

Both injections in COP (GR03-C1 and 981-C2) resulted in patterns of cell labeling that differed from the PMD cases. In both cases, labeled cells were mostly found in the ventrolateral aspect of the nucleus (within the parvocellular and subtrigeminal subdivisions) with a more prominent involvement of the caudal onehalf of contralateral LRn. However, there were no labeled cells in the linear subdivision. Like the $\mathrm{C} 1$ and $\mathrm{C} 2$ cases in PMD, some zonal differences may also be present because the caudal tip of the nucleus was more heavily involved in GR03 (a C1 zone case) compared with case 981 (a C2 zone case).

A comparison of the number and laterality of labeled neurons observed within the Pn and LRn is presented in Figure 8 (also see Table 1). Figure $8 \mathrm{~A}$ indicates the relationship between numbers of labeled cells in the inferior olive and those within LRn and Pn. The numbers of neurons labeled within the LRn and inferior olive are positively correlated $(r=0.87 ; p<0.005)$. Assuming, for different cases, a similar spread of tracer within the molecular layer and subjacent granular layer and a uniform projection density for climbing fibers (cf. Herrero et al., 2002), this suggests that the contribution from LRn is mostly related to the size of the injection site rather than to the identity of the injected cortical lobule or zone. However, when labeled cell numbers in Pn and the olive were compared, no significant correlation was noted $(r=0.47 ; p>0.05)$, implying that other factors such as cortical (zonal) location may be more important factors than injection site size in determining involvement of Pn neurons. This is supported by the finding that cases with an injection in the D0/D1 zone in PMD have a significantly higher Pn/LRn ratio compared with either $\mathrm{C} 2$ or $\mathrm{C} 1$ injections in PMD or COP (for both comparisons: two-sided $t$ test assuming unequal variances, $p<0.05$ ). A significantly higher $\mathrm{Pn} / \mathrm{LRn}$ ratio was also found for the $\mathrm{C} 2$ zone group of cases with respect to the $\mathrm{C} 1$ zone group (two-sided $t$ test, $p<0.05)$. Finally, no obvious distinction could be found in the numbers of labeled neurons located in ipsilateral or contralateral LRn (Fig. 8C). For the Pn, however, the number of ipsilaterally labeled neurons tended to decrease when the injection was placed in more lateral zones (i.e., resulting in a higher contralateral/ipsilateral ratio) (Fig. $8 D$ ). An exception is presented by case GR03 in which the $\mathrm{C} 1$ zone injection in COP resulted in $\sim 40$ times as many cells in the contralateral Pn as in the ipsilateral Pn. This contrasts with the more prominent bilateral nature of the LRn projection in this case.

\section{Discussion}

The present study makes four key observations regarding interconnectivity between functionally related parts of the cerebellar cortex: (1) mossy fiber collaterals mostly distribute to the same
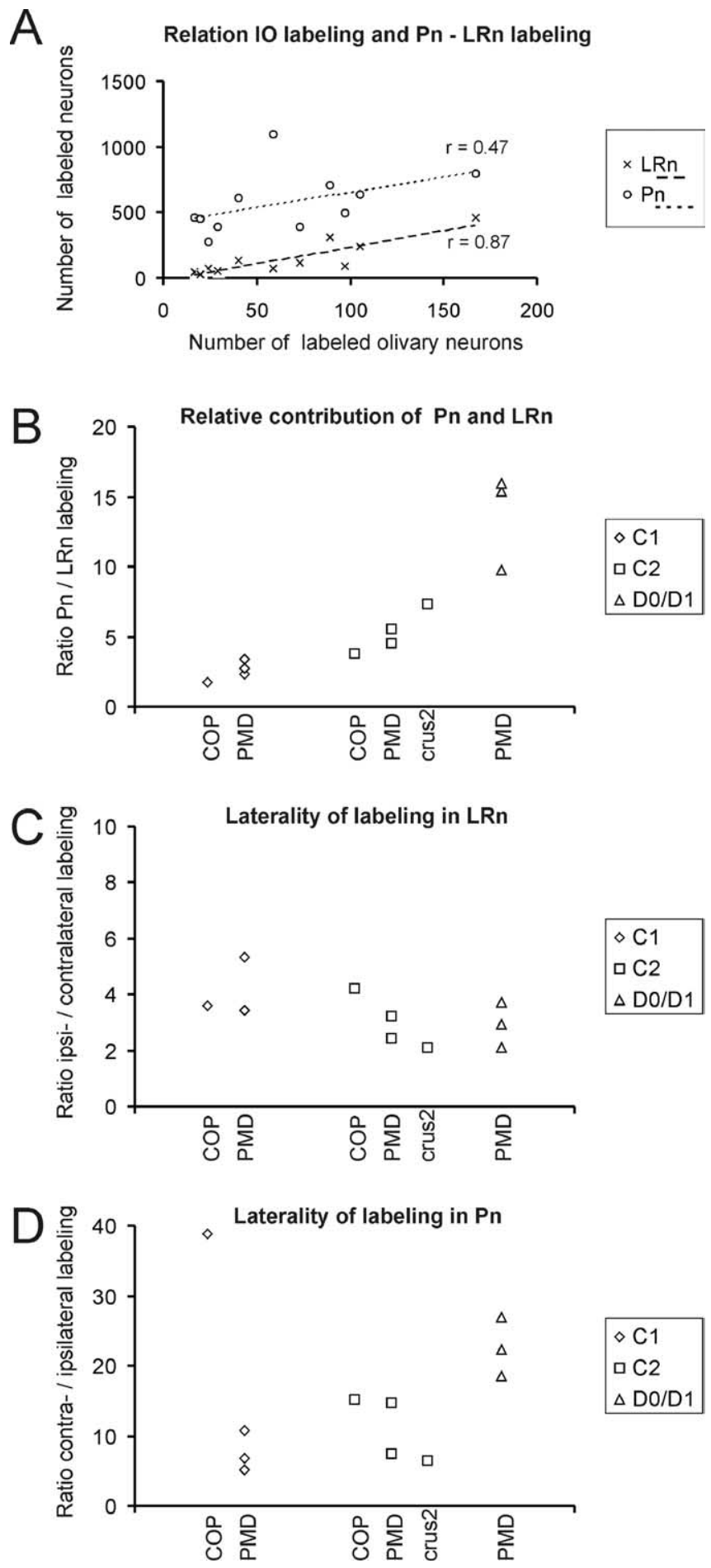

Figure 8. A comparison of the number and laterality of labeled neurons observed within the Pn and LRn. A, Graph depicting the relationship between number of labeled olivary neurons and number of labeled neurons in $P n$ and LRn. Note that the numbers of labeled LRn and IO neurons correlate better than the numbers of labeled $\mathrm{Pn}$ and $\mathrm{IO}$ neurons, indicating that the involvement of both precerebellar centers to provide mossy fiber afferents to the granular layer subjacent a particular climbing fiber zone differs from each other. $\boldsymbol{B}$, Graph depicting the contribution of $\mathrm{Pn}$ and LRn to a particular injection site. Note that injections into the DO/D1 zone of PMD results in a much higher ratio of labeled neurons in $\mathrm{Pn}$ and LRn than in cases in which the injection was centered on C1. C, D, Graphs comparing the participation of ipsilaterally versus contralaterally labeled neurons in $\operatorname{Ln}(\boldsymbol{C})$ and $\mathrm{Pn}(\boldsymbol{D})$. Note that, for the $L R n$, the ratio of the number of ipsilaterally and contralaterally labeled neurons varies only marginally between different cases, whereas notable differences are observed for the Pn. Data for Pn labeling are in part taken from Pijpers and Ruigrok (2006). 
cerebellar lobules as climbing fiber collaterals; (2) collaterals of labeled climbing fibers are always accompanied by labeled mossy fiber terminals in the subjacent granular layer; (3) additional mossy fiber collaterals terminate in specific, nonadjacent, striplike regions, which usually have the same zebrin signature as the source of the collateralization; and (4) mossy fiber projections to different cortical zones are accompanied by significant differences in projection densities from two major brainstem sources. The present results therefore indicate that the mossy fiber and climbing fiber afferent systems are closely aligned and that this is a consistent and widespread feature of cerebellar cortical organization in the rat.

Because of its uniform structure, functional localization within the cerebellar cortex critically depends on the organization of its afferent systems (for review, see Manni and Petrosini, 2004). On the one hand, mossy fiber input is usually characterized by multiple representations of the same body parts distributed as a mosaic of patches within the cerebellar cortex [the fractionated somatotopy of Welker (1987)]; however, note that some aspects of mossy fiber organization have also been described in terms of longitudinal strips (Ekerot and Larson, 1980; Serapide et al., 2001). In contrast, climbing fiber inputs with similar receptive field characteristics adhere to parasagittally arranged zones in which finer microzonal subdivisions can be recognized (Oscarsson, 1979; Apps and Garwicz, 2005). Perhaps not surprisingly, then, physiological studies disagree on the extent to which the somatotopic pattern of mossy and climbing fibers are aligned to each other (Garwicz et al., 1998; Brown and Bower, 2001). Resolving this disagreement is important for understanding basic cerebellar processing (Apps and Garwicz, 2005), and the present study helps inform this debate by providing the most comprehensive description to date of the distribution of functionally related climbing and mossy fiber collaterals.

\section{Cerebellar somatotopy and collateralization of mossy and climbing fiber afferents}

Climbing fiber somatotopic representations of the same body parts have been recognized in regions of the anterior as well as within the posterior cerebellum (for reviews, see Manni and Petrosini, 2004; Apps and Garwicz, 2005). In rat, at the zonal level, representations of the ipsilateral forelimb are present in the C1 and C3 zones of PMD and SL, whereas bilateral forelimb representations are present in the $\mathrm{C} 2$ zone of the same lobules (Atkins and Apps, 1997; Pardoe and Apps, 2002). Likewise, COP harbors zones that are characterized by ipsilateral (C1) and bilateral (C2) climbing fiber inputs from the hindlimb (Atkins and Apps, 1997), and these body representations are likely to have counterparts in the anterior lobe (Jorntell et al., 2000). The anatomical substrate for this homology has been demonstrated by Voogd et al. (2003), and was confirmed in the present study. Indeed, climbing fibers collateralize from zones in PMD and COP to target similar zones in SL and anterior lobe, respectively (Pardoe and Apps, 2002). Here, we extend this finding by showing that mossy fiber collaterals, labeled from the same injection site, also exhibit a similar somatotopic distribution. Collaterals labeled from COP mostly reach the anterior lobe, whereas PMD injections result in labeled mossy fibers within SL and the crura. The present findings therefore strongly imply that these particular cerebellar regions (COP and the anterior lobe; PMD, SL, and the crura) function in close association with each other.

The distribution of mossy fiber collaterals, however, was always more widespread than the climbing fiber collaterals arising from the same injection site, being clustered within multiple, essentially longitudinal patterns within vermal, paravermal, and hemispheral regions of the targeted lobules. These patterns are usually mimicked contralaterally with the notable exception of patches located in the lateral-most parts of the ipsilateral cortex. The former suggests that the same information conveyed via the mossy fiber system targets a number of different cortical zones bilaterally, perhaps for the regulation of whole-body movements. The latter suggests that lateral zones are more concerned with unilateral inputs.

\section{Collateralization and zebrin pattern}

The relationship between the organization of the olivocerebellar climbing fiber system and the zebrin pattern of Purkinje cells has recently been described in considerable detail (Sugihara and Shinoda, 2004; Voogd and Ruigrok, 2004; Pijpers et al., 2005). Using $2 \mathrm{D}$ and $3 \mathrm{D}$ reconstruction methods and quantitative analysis, the present observations significantly extend these previous findings by showing that the distribution of subpopulations of mossy fiber rosettes with a common lobular and zonal target (but with heterogeneous origin), also show close relationships to that of the overlying zebrin pattern. If the rather weakly positive zebrin patch "e+" of COP and zone P3+ of the anterior lobe are considered as part of a zebrin-negative territory (see Results), then mossy fiber collaterals labeled from injections confined to the $\mathrm{C} 1$ zone preferentially target regions of granular layer subjacent to zebrin-negative Purkinje cells, whereas C2 zone injections result in labeled mossy fiber rosettes below zebrin-positive regions (Gravel and Hawkes, 1990; Ji and Hawkes, 1994), Our observations therefore underscore the functional differentiation of zebrin-negative versus zebrin-positive Purkinje cells (Voogd et al., 2003; Sugihara and Shinoda, 2004; Voogd and Ruigrok, 2004) and suggest that the organization of the granular layer correlates to this patterning. Interestingly, some studies have indicated that zebrin-positive and -negative Purkinje cells may operate differently (Nagao et al., 1997; Wadiche and Jahr, 2005).

\section{Somatotopy, zones, and origin of mossy fibers}

Mossy fibers terminating in COP and PMD convey somatosensory information from the periphery by way of the spinal cord, dorsal column nuclei, trigeminal nuclei, and/or LRn. In addition, preprocessed information from the cerebral cortex may reach these areas via pontine relays (for review, see Ruigrok, 2004). Consequently, every $\mathrm{CTb}$ injection site will result in retrogradely labeled neurons located within a specific combination of these precerebellar centers. We have concentrated our analysis on the LRn and the Pn (Pijpers and Ruigrok, 2006) and show that the distribution of neurons within both structures correlates closely with the lobular identity of the injection site. However, at best only subtle differences were noted in the distribution of labeled neurons arising from injections targeted at different zones within the same lobule. Because the collateral distribution of mossy fibers is strongly affected by the identity of the injected zone (see below), this suggests that either (1) cells within LRn and Pn projecting to different zones are intermingled in each structure or (2) a subnuclear topical organization is present that could not be detected in the present experiments. The clearest differences we found related to the proportion of retrogradely labeled neurons arising from Pn as compared with LRn targeting different zones. These differences suggest at least a partly independent origin of mossy fiber terminals to the $\mathrm{C} 1, \mathrm{C} 2$, and D1/D0 zones. Thus, highly specific combinations of mossy fibers appear to distribute terminals to particular lobules and zones of the cerebellar cortex. Future experiments using multiple tracer techniques will be re- 
quired to determine whether the observed zonal differences in collateralization patterns relate to any differences in topography in projections arising from the various precerebellar sources.

\section{Congruence of climbing and mossy fiber collaterals}

Physiologically, convergence of mossy and climbing fiber receptive fields was first demonstrated by Eccles and collaborators (Eccles et al., 1968; Kitai et al., 1969). More recently, micromapping techniques have also focused on the importance of convergence of both afferent systems. Indeed, both for cat forelimb (Garwicz et al., 1998; Apps and Garwicz, 2005) as well as for rat perioral regions (Brown and Bower, 2001) a general similarity of the receptive fields of mossy fibers and climbing fibers has been noted in localized regions of the cerebellar cortex. The present data provide evidence of the anatomical substrate for this functional congruence. However, the functional implications of such a related distribution of mossy and climbing fibers remains to be resolved [for example, see Ekerot and Jorntell (2001) compared with Brown and Bower (2001); Lu et al. (2005)].

\section{Concluding remarks}

The present study shows that labeled mossy fiber rosettes closely accompany collaterals of climbing fibers labeled from the same zonal location. A similar, but more tentative observation was made by Voogd et al. (2003) and was also mentioned for the vestibulocerebellum (Ruigrok, 2003). Such an arrangement implies that the specific combination of mossy and climbing fiber inputs processed at a given locus within the cerebellar cortex (i.e., within PMD or COP) may be repeated (at least in part) in other cerebellar regions (such as SL or anterior lobe). This finding is consistent with the idea that the cerebellum is modular and that basic operational units are formed by multizonal microcomplexes (i.e., two or more microzones within different parts of the cortex with common climbing fiber input have output to a common group of cerebellar nuclear neurons) (Apps and Garwicz, 2005). Although, originally, this was thought to enable parallel processing of information derived from different combinations of mossy fibers, the present results extend this hypothesis to suggest that microcomplexes also make use of some common mossy fiber inputs.

\section{References}

Albus JS (1971) A theory on cerebellar function. Math Biosci 10:25-61.

Alisky JM, Tolbert DL (1997) Quantitative analysis of converging spinal and cuneate mossy fibre afferent projections to the rat cerebellar anterior lobe. Neuroscience 80:373-388.

Apps R, Garwicz M (2005) Anatomical and physiological foundations of cerebellar information processing. Nat Rev Neurosci 6:297-311.

Atkins MJ, Apps R (1997) Somatotopical organisation within the climbing fibre projection to the paramedian lobule and copula pyramidis of the rat cerebellum. J Comp Neurol 389:249-263.

Brochu G, Maler L, Hawkes R (1990) Zebrin II: a polypeptide antigen expressed selectively by Purkinje cells reveals compartments in rat and fish cerebellum. J Comp Neurol 291:538-552.

Brown IE, Bower JM (2001) Congruence of mossy fiber and climbing fiber tactile projections in the lateral hemispheres of the rat cerebellum. J Comp Neurol 429:59-70.

Chen S, Aston-Jones G (1998) Axonal collateral-collateral transport of tract tracers in brain neurons: false anterograde labelling and useful tool. Neuroscience 82:1151-1163.

Eccles JC, Provini L, Strata P, Taborikova H (1968) Analysis of electrical potentials evoked in the cerebellar anterior lobe by stimulation of hindlimb and forelimb nerves. Exp Brain Res 6:171-194.

Ekerot CF, Jorntell H (2001) Parallel fibre receptive fields of Purkinje cells and interneurons are climbing fibre-specific. Eur J Neurosci 13:1303-1310.
Ekerot CF, Larson B (1980) Termination in overlapping sagittal zones in cerebellar anterior lobe of mossy and climbing fiber paths activated from dorsal funiculus. Exp Brain Res 38:163-172.

Garwicz M, Jorntell H, Ekerot CF (1998) Cutaneous receptive fields and topography of mossy fibres and climbing fibres projecting to cat cerebellar C3 zone. J Physiol (Lond) 512:277-293.

Gravel C, Hawkes R (1990) Parasagittal organization of the rat cerebellar cortex: direct comparison of Purkinje cell compartments and the organization of the spinocerebellar projection. J Comp Neurol 291:79-102.

Hawkes R, Leclerc N (1987) Antigenic map of the rat cerebellar cortex: the distribution of parasagittal bands as revealed by monoclonal antiPurkinje cell antibody mabQ113. J Comp Neurol 256:29-41.

Herrero L, Pardoe J, Apps R (2002) Pontine and lateral reticular projections to the $\mathrm{cl}$ zone in lobulus simplex and paramedian lobule of the rat cerebellar cortex. Cerebellum 1:185-199.

Ito M (1984) The cerebellum and neural control. New York: Raven.

Ito M (2006) Cerebellar circuitry as a neuronal machine. Prog Neurobiol 78:272-303.

Ji Z, Hawkes R (1994) Topography of Purkinje cell compartments and mossy fiber terminal fields in lobules II and III of the rat cerebellar cortex: spinocerebellar and cuneocerebellar projections. Neuroscience 61:935-954.

Jorntell H, Ekerot C, Garwicz M, Luo XL (2000) Functional organization of climbing fibre projection to the cerebellar anterior lobe of the rat. J Physiol (Lond) 522:297-309.

Kitai ST, Taborikova H, Tsukahara N, Eccles JC (1969) The distribution to the cerebellar anterior lobe of the climbing and mossy fiber inputs from the plantar and palmar cutaneous afferents. Exp Brain Res 7:1-10.

Lu H, Hartmann MJ, Bower JM (2005) Correlations between Purkinje cell single unit activity and simultaneously recorded field potentials in the immediately underlying granule cell layer. J Neurophysiol 94:1849-1860.

Manni E, Petrosini L (2004) A century of cerebellar somatotopy: a debated representation. Nat Rev Neurosci 5:241-249.

Marr D (1969) A theory of cerebellar cortex. J Physiol (Lond) 202:437-470.

Mihailoff G (1993) Cerebellar nuclear projections from the basilar pontine nuclei and nucleus reticularis tegmenti pontis as demonstrated with PHA-L tracing in the rat. J Comp Neurol 330:130-146.

Nagao S, Kwak S, Kanazawa I (1997) EAAT4, a glutamate transporter with properties of a chloride channel, is predominantly localized in Purkinje cell dendrites, and forms parasagittal compartments in rat cerebellum. Neuroscience 78:929-933.

Newman DB, Ginsberg CY (1992) Brainstem reticular nuclei that project to the cerebellum in rats: a retrograde tracer study. Brain Behav Evol 39:24-68.

Odeh F, Ackerley R, Bjaalie JG, Apps R (2005) Pontine maps linking somatosensory and cerebellar cortices are in register with climbing fiber somatotopy. J Neurosci 25:5680-5690.

Oscarsson O (1979) Functional units of the cerebellum — sagittal zones and microzones. Trends Neurosci 2:143-145.

Pardoe J, Apps R (2002) Structure-function relations of two somatotopically corresponding regions of the rat cerebellar cortex: olivo-corticonuclear connections. Cerebellum 1:165-184.

Pijpers A, Ruigrok TJH (2006) Organization of pontocerebellar projections to identified climbing fiber zones in the rat. J Comp Neurol 496:513-528.

Pijpers A, Voogd J, Ruigrok TJH (2005) Topography of olivo-corticonuclear modules in the intermediate cerebellum of the rat. J Comp Neurol 492:193-213.

Ruigrok TJ (2003) Collateralization of climbing and mossy fibers projecting to the nodulus and flocculus of the rat cerebellum. J Comp Neurol 466:278-298.

Ruigrok TJ, Voogd J (2000) Organization of projections from the inferior olive to the cerebellar nuclei in the rat. J Comp Neurol 426:209-228.

Ruigrok TJH (1997) Cerebellar nuclei: the olivary connection. In: The cerebellum: from structure to control (De Zeeuw CI, Strata P, Voogd J, eds), pp 162-197. Amsterdam: Elsevier Science.

Ruigrok TJH (2004) Precerebellar nuclei and red nucleus. In: The rat nervous system, Ed 3 (Paxinos G, ed), pp 167-204. San Diego: Elsevier Academic.

Ruigrok TJH, Teune TM, van der Burg J, Sabel-Goedknegt H (1995) A retrograde double labeling technique for light microscopy. A combination of axonal transport of cholera toxin B-subunit and a gold-lectin conjugate. J Neurosci Methods 61:127-138. 
Serapide MF, Panto MR, Parenti R, Zappala A, Cicirata F (2001) Multiple zonal projections of the basilar pontine nuclei to the cerebellar cortex of the rat. J Comp Neurol 430:471-484.

Sugihara I, Shinoda Y (2004) Molecular, topographic, and functional organization of the cerebellar cortex: a study with combined aldolase $\mathrm{C}$ and olivocerebellar labeling. J Neurosci 24:8771-8785.

Sultan F (2001) Distribution of mossy fibre rosettes in the cerebellum of cat and mice: evidence for a parasagittal organization at the single fibre level. Eur J Neurosci 13:2123-2130.

Teune TM, van der Burg J, De Zeeuw CI, Voogd J, Ruigrok TJH (1998) Single Purkinje cell can innervate multiple classes of projection neurons in the cerebellar nuclei of the rat: a light microscopic and ultrastructural triple-tracer study in the rat. J Comp Neurol 392:164-178.

Voogd J, Bigaré F (1980) Topographical distribution of olivary and cortico nuclear fibers in the cerebellum: a review. In: The inferior olivary nucleus. Anatomy and physiology (Courville J, de Montigny C, Lamarre Y, eds), pp 207-234. New York: Raven.

Voogd J, Glickstein M (1998) The anatomy of the cerebellum. Trends Neurosci 2:305-371.

Voogd J, Ruigrok TJ (2004) The organization of the corticonuclear and olivocerebellar climbing fiber projections to the rat cerebellar vermis: the congruence of projection zones and the zebrin pattern. J Neurocytol 33:5-21.

Voogd J, Pardoe J, Ruigrok TJ, Apps R (2003) The distribution of climbing and mossy fiber collateral branches from the copula pyramidis and the paramedian lobule: congruence of climbing fiber cortical zones and the pattern of zebrin banding within the rat cerebellum. J Neurosci 23:4645-4656.

Wadiche JI, Jahr CE (2005) Patterned expression of Purkinje cell glutamate transporters controls synaptic plasticity. Nat Neurosci 8:1329-1334.

Welker W (1987) Spatial organization of somatosensory projections to granule cell cerebellar cortex: functional and connectional implications of fractured somatotopy (summary of Wisconsin studies). In: New concepts in cerebellar neurobiology (King JS, ed), pp 239-280. New York: Alan R. Liss.

Wu HS, Sugihara I, Shinoda Y (1999) Projection patterns of single mossy fibers originating from the lateral reticular nucleus in the rat cerebellar cortex and nuclei. J Comp Neurol 411:97-118.

Yatim N, Billig I, Compoint C, Buisseret P, Buisseret-Delmas C (1996) Trigeminocerebellar and trigemino-olivary projections in rats. Neurosci Res 25:267-283. 